\title{
Schedule Optimization in a Smart Microgrid Considering Demand Response Constraints
}

\author{
Julian Garcia-Guarin ${ }^{1}\left(\mathbb{C}\right.$, David Alvarez ${ }^{1}\left(\mathbb{C}\right.$, Arturo Bretas $^{2}\left(\mathbb{D}\right.$ and Sergio Rivera ${ }^{1,2, *(\mathbb{C}}$ \\ 1 Electrical and Electronics Engineering Department, Engineering Faculty, Universidad Nacional de Colombia, \\ Bogotá 111321, Colombia; pjgarciag@unal.edu.co (J.G.-G.); dlalvareza@unal.edu.co (D.A.) \\ 2 Engineering Faculty, University of Florida, Gainesville, FL 32611, USA; arturo@ece.ufl.edu \\ * Correspondence: srriverar@unal.edu.co; Tel.: +57-1-320-463-2806
}

Received: 23 July 2020; Accepted: 1 September 2020; Published: 3 September 2020

check for updates

\begin{abstract}
Smart microgrids (SMGs) may face energy rationing due to unavailability of energy resources. Demand response (DR) in SMGs is useful not only in emergencies, since load cuts might be planned with a reduction in consumption but also in normal operation. SMG energy resources include storage systems, dispatchable units, and resources with uncertainty, such as residential demand, renewable generation, electric vehicle traffic, and electricity markets. An aggregator can optimize the scheduling of these resources, however, load demand can completely curtail until being neglected to increase the profits. The DR function (DRF) is developed as a constraint of minimum size to supply the demand and contributes solving of the $0-1$ knapsack problem (KP), which involves a combinatorial optimization. The 0-1 KP stores limited energy capacity and is successful in disconnecting loads. Both constraints, the 0-1 KP and DRF, are compared in the ranking index, load reduction percentage, and execution time. Both functions turn out to be very similar according to the performance of these indicators, unlike the ranking index, in which the DRF has better performance. The DRF reduces to $25 \%$ the minimum demand to avoid non-optimal situations, such as non-supplying the demand and has potential benefits, such as the elimination of finite combinations and easy implementation.
\end{abstract}

Keywords: load shedding; optimization of energy demand supply; smart microgrid scheduling; 0-1 knapsack problem

\section{Introduction}

User participation has become of great value in smart grids management. Cooperation among users allows decision-making more flexibility, through the use of demand response (DR). Customers can be included in DR programs either by changing their habits or by implementing load control [1]. DR can be analyzed from two approaches: the first considers the power quality that is affected by disturbances, such as harmonics, inter-harmonics, phase unbalance, phase jump, and temperature effects due to overloads [2,3]. The second encompasses demand benefits for reducing operating costs, improving aggregator profits, and mitigating market power [3,4]. This research falls under the second group, which saves energy through demand management [5].

Demand management can consider demand forecasts, load curtailment, and combinatorial optimization with DR [6,7]. This type of combinatorial optimization problem has several applications for load shedding [8]. Heuristic techniques have been applied to solve this problem with non-deterministic polynomial times, also called NP-hard problems [8,9]. This type of problem can arise as a subproblem or a constraint $[5,8,9]$. The solution was presented as a combination of a series of user decisions $[5,8,9]$. In microgrids, load shedding was modeled through the 0-1 knapsack problem (KP), which is classified as an NP-hard problem [8]. In using this technique, the possibilities and solution times are exponentially increased with $2^{\mathrm{N}}$ [8]. The $0-1 \mathrm{KP}$ problem was solved with heuristic algorithms and Lagrangian 
multipliers. For example, Cuckoo Search and Tabu Search are heuristic algorithms that are applied efficiently by solving multidimensional $0-1 \mathrm{KP}[8,10]$. In addition, the method of Lagrangian multipliers is implemented with integer programming. Results show that execution time is polynomial and is similar to other findings [11].

Load restoration has been addressed with 0-1 KP in microgrids. Consumer supply is an important procedure after the smart microgrid (SMG) is out of service [12,13]. For example, failures and blackouts can deteriorate customer satisfaction and restoration of power supply is essential [12,13]. DR programs share the objectives of ensuring user welfare and supplying essential loads [14]. Energy must be supplied at low cost and supplying critical loads $[12,13]$. The energy supply is optimized through heuristic techniques $[12,13]$. Consequently, DR programs are supported by load restoration programs, in both cases the aim is to ensure the energy supply of a group of users.

Optimization techniques with heuristics have been widely studied [15]. SMGs comprise interacting elements such as residential loads with DR programs, distributed electric vehicles (EVs), energy storage systems (ESSs), generation with renewable energies, and dispatchable units [13,14]. SMGs may also be subject to sources of uncertainty that further complicate operation, such as residential demand, renewable generation, traffic of EVs, and electricity markets $[13,15]$. For example, a comparative analysis is performed between various algorithms for a SMG model $[9,16]$. The variable neighborhood search-differential evolutionary particle swarm optimization (VNS-DEEPSO) algorithm turned out to be better than the chaotic evolutionary particle swarm optimization (PSO), differential evolution (DE) with stochastic selection, enhanced velocity differential evolutionary PSO, firefly, improved chaotic DEEPSO, improved DE, PSO with global best perturbation, and unified PSO algorithms [16]. Research on these algorithms suggests implementing constraints for DR [17-19].

\subsection{Motivation of This Paper}

Operational costs optimization with heuristics alleviates renewable energy disadvantages such as intermittency and fluctuations that can be addressed with the management of ESSs. Heuristics with probabilistic analysis provide robust solutions to the uncertainty of renewables [20]. In addition, the energy supply is considered as an additional objective under criteria of frequency, duration, and magnitude $[4,20]$. For example, the reduction of operational costs and energy supply are considered in multi-objective optimization problems, this approach presents as a drawback of multiple optimal solutions on the Pareto front $[4,20]$. However, in the traditional approach the problem is addressed considering 0-1 KP restrictions, however the polynomial execution times make it difficult to analyze real microgrids [14]. Solving problems involving polynomial execution times, robust solutions in SMGs with uncertainty, and addressing multiple criteria in a single objective function motivates the findings of this research.

\subsection{Contribution of This Paper}

This article presents an implementation of 0-1 KP constraints for DR. In addition, the DR function (DRF) is developed as a contribution of this research that solves the 0-1 KP, which indeed involves combinatorial optimization. Both techniques, DRF and 0-1 KP, are evaluated in a SMG model considering an aggregator that seeks social welfare and supplies essential loads. The aggregator schedules resources with or without uncertainty. The uncertainty resources are EVs trips, renewable generation, loads with DR, and energy market prices [21]. The resources without uncertainty are distributed generators (DGs) and ESSs. Additionally, this article makes the following contributions:

(1) The implementation of 0-1 KP in a SMG model. 0-1 KP is formulated in two levels. In the first level the demand is grouped, and in the second level the demand is discretized into hour blocks. The refinement of the blocks depends on the computing capacity. Results are measured in terms of the ranking index (RI), load reduction percentage, and execution time. The RI is calculated as the sum of the average profits and their standard deviation. 
(2) DRF is developed to solve the 0-1 KP. DRF is compared with 0-1 KP in terms of the RI, load reduction percentage, and execution time. The outstanding outcomes of DRF are similar to those of the 0-1 KP. However, DRF stands out for the following characteristics. DRF works with continued variables, so DRF has no problem of refinement. In addition, DRF needs no additional execution time for preloading combinations, since the polynomial time in $0-1 \mathrm{KP}$ and its function are easier to implement than 0-1 KP.

The article presents the following structure: Section 2 summarizes the state of the art, Section 3 presents the SMG model, Section 4 formulates 0-1 KP and DRF, Section 5 contains the results, and Section 6 presents the main conclusions of the research.

\section{State of the Art}

Table 1 shows the SMG models with energy resources that are subject to $0-1 \mathrm{KP}$ and DRF restrictions [18-26]. These models are listed from 1 to 9 and are described in the following. The SMG model 1 considers a maximum generation capacity at $0-1 \mathrm{KP}$ and is solved by using binary variables. The SMG model 1 also considers the maximization of the benefits of SMG [14]. The 0-1 KP solution is successful. However, this model has the problem of polynomial times. Since the SMG model 1 represents a simple microgrid, it lacks elements such as load with DR, market prices, EVs, and ESSs. Therefore, the implementation of the SMG model 1 is feasible in didactic and short-range scenarios of actual SMGs [14].

The SMG model 2 gathers elements such as DGs, photovoltaic (PV) generation, external suppliers, load with DR, market prices, EVs, and ESSs $[13,17,27,28]$. The SMG model 2 also has sources of uncertainty such as residential demand, renewable generation, traffic of EVs, and electricity markets $[13,17,27]$. The model is based on the operation of a residential microgrid [16]. However, the residential customer demand is unattended after the optimization process, that is, the load demand is close to zero. In demand management, this is an unwanted solution [12,16]. The SMG model 2 is improved with a battery swapping station for EVs and sets a DRF as a constraint [22]. However, DRF is a target rather than a constraint in [4]. Therefore, the new implementation of the DRF lacks a previous study for the implementation with loads with DR.

Table 1. Review of microgrids energy resources with 0-1 knapsack problem (KP) and demand response function (DRF).

\begin{tabular}{cccccccl}
\hline No. & Gen & ESS & DR & EVs & $\mathbf{0 - 1}$ KP & DRF & \\
\hline 1 & Yes & No & Yes & No & Yes & No & Not reported [14] \\
2 & Yes & Yes & Yes & Yes & No & Yes & EVs, renewable resources, electricity markets, and loads with DR [22] \\
3 & No & No & Yes & No & No & No & Electricity markets and loads with DR [23] \\
4 & Yes & Yes & No & No & No & No & Renewable resources and loads [24] \\
5 & Yes & Yes & Yes & No & No & No & Renewable resources, loads, and market prices [25] \\
6 & Yes & Yes & No & Yes & No & No & Renewable resources, loads, and EVs [26] \\
7 & Yes & Yes & Yes & No & No & No & Renewable resources, loads, and market prices [27] \\
8 & Yes & Yes & No & No & No & No & Not reported [29] \\
9 & Yes & No & No & No & No & No & Not reported [30,31] \\
10 & Yes & Yes & Yes & Yes & Yes & Yes & EVs, renewable resources, electricity markets, and loads with DR \\
& & & & & & &
\end{tabular}

The residential power system model 3 aims to improve the economic benefits [23]. This model considers the use of smart meters in SMGs [1] as well as load uncertainty and price volatility in real time [23]. This model has no restrictions in optimization, which ensures a good state of the demand, such as 0-1 KP and DRF [23]. The analysis does not consider EVs and ESSs [23]. The power system model 4 takes into account the predictive forecast but not load shedding [24]. In addition, batteries and generation with combined heat and power and renewable are considered to increase profits [24]. This model includes neither DR programs nor EVs [24].

The model 5 of distributed resources supplies the demand side with renewable energy [25] and aims to reduce $\mathrm{CO}_{2}$ emissions and energy costs. The technique restricts the energy demand supplying 
and is similar to $0-1 \mathrm{KP}$, but in this case seven probability scenarios are studied. These seven scenarios are obtained by using a scenario reduction technique. This model is strictly limited to plausible scenarios in uncertain environments; therefore, it can find plausible but not optimal solutions [25].

The smart grid model 6 comprises EVs, ESSs, and renewable generation. This model aims to reduce operating costs and lower $\mathrm{CO}_{2}$ emissions from thermal power plants [26]. This model predicts the load; however, it warns of deviations from the actual load. The model includes no implementation of load with DR [26]. The small-scale model 7 of smart grid aims to maximize the profits in the grid and considers a system with DGs, renewable energies, and ESSs [27]. In this model, the loads are represented by an agent that controls the electric power and exchanges information with other agents in the network [27]. The major drawback of this formulation is that the model lacks load demand management strategies and is difficult to reproduce. Nevertheless, it provides viable results despite having no load restrictions.

The microgrid model 8 reduces operation costs while the dispatchable generation units and the storage of energy are scheduled. Errors for forecasting demand in the operation are recommended to be studied in future works. The microgrid model 8 highlights operations to optimize costs, such as load curtailment and load shifting [29]. Power network models in 9 analyze generation costs, power losses, emissions, and validate an evolutionary hybrid algorithm. This research has demonstrated the interest of the scientific community in validating optimization algorithms with multiple tests. However, the study neglects sources of uncertainty, ESSs, VEs, loads with response to demand, and penalties for not supplying the demand [32]. Table 2 summarizes the limitations presented in the review of this section.

The SMG model 10 is implemented in this research and overcomes some drawbacks described in previous literature. First, this model addresses residential loads with DR programs, EVs, ESSs, DGs, and renewable resources. An aggregator aims to increase profits and can negotiate the buy/sell energy in electricity markets. The microgrid considers uncertainty conditions that are more challenging in the operation, such as renewable generation forecasts, trip planning with EVs, market price volatility, and load forecast.

Table 2. Limitations of the review of microgrids with 0-1 KP and DRF.

\begin{tabular}{|c|c|}
\hline No. & Limitations of the Review of Microgrids Energy Resources with 0-1 KP and DRF. \\
\hline 1 & The model 1 lacks elements such as load with DR, market prices, EVs, and ESSs [14]. \\
\hline 2 & $\begin{array}{l}\text { The residential customer demand is unattended after the optimization process. In demand management, this is an } \\
\text { unwanted solution [22]. }\end{array}$ \\
\hline 3 & The model 3 has no restrictions in optimization, which ensures a good state of the demand, such as 0-1 KP and DRF [23]. \\
\hline 4 & The model 4 does not include either DR programs or EVs [24]. \\
\hline 5 & $\begin{array}{l}\text { The model } 5 \text { is strictly limited to plausible scenarios in uncertain environments; therefore, it can find feasible but not } \\
\text { optimal solutions [25]. }\end{array}$ \\
\hline 6 & The model 6 includes no implementation of load with DR [26]. \\
\hline 7 & $\begin{array}{l}\text { The major drawback of this formulation is that the model } 7 \text { lacks load demand management strategies and is difficult to } \\
\text { reproduce [27]. }\end{array}$ \\
\hline 8 & Errors for forecasting demand in the operation are recommended to be studied in future works [29]. \\
\hline 9 & The study neglects sources of uncertainty, ESSs, VEs, loads with DR, and penalties for not supplying the demand [31]. \\
\hline 10 & Model 10 overcomes the above limitations. \\
\hline
\end{tabular}

The SMG model 10 overcomes the weaknesses mentioned in previous models, such as ensuring that the demand is met, integrating DR programs, generating a feasible SMG model for optimization, and creating a reproducible method. This model includes 0-1 KP and DRF to optimize the loads with DR. The $0-1 \mathrm{KP}$ implementation consists of discretizing the load for its optimization and guaranteeing that the demand is satisfied. DRF is a constraint in the objective function to ensure welfare of the demand. This model is the most complete according to the literature review shown in Table 1 that includes sources of uncertainty, elements of the SMG, and 0-1 KP and DRF demand restrictions.

Various heuristic algorithms have been studied to solve SMG models. The resulting VNS-DEEPSO algorithm has the highest performance of the algorithms mentioned above according to [12,16]. The mechanisms implemented in this heuristic are described below. 


\subsection{VNS-DEEPSO Algorithm}

This technique assembles the VNS algorithm and the DEEPSO algorithm [12,16]. VNS-DEEPSO algorithm solves an SMG optimization problem with uncertainty, proposed by the GECAD group in [16]. The VNS-DEEPSO secured the first place in this world competition [16,19], as their algorithm considerably improved the results obtained with the differential evolution (DE)/rand/1 algorithm by $279 \%$ in a case study with uncertain environments [30]. This study determined an optimal participation of VNS and DEEPSO of $48.6 \%$ and $51.4 \%$, respectively [30]. In the early stages, VNS searches for neighborhood structures, scans distant neighborhoods, and maintains the most current solution. The solution is improved with local search method in two steps. In the first step, the cyclic coordinate method searches for a set of directions to optimize non-differentiable or nonlinear functions. In the second step, the Fibonacci line search method reduces the uncertainty interval, as shown in Figure 1a [33]. DEEPSO has the structure of PSO. The new particles are calculated by calculating (1) the new velocity of the particle, (2) the new position, and (3) the particle mutation weight, as shown in Figure 1b. Additionally, the particles change their position inspired by DE and evolutionary algorithms. The improvements include noise to affect the particle positions, thereby adding positive benefits. Moreover, the movement of memory is replaced by the movement of perception. This improvement aims to track the direction of the local gradient. These improvements lead to a solution with remarkable convergence [34]. The pseudocode and link of the complete code are presented in Figure 1 for the VNS and DEEPSO algorithms [16,19].

\subsection{Conceptual Formulation}

The conceptual formulation is based on the flow diagram as shown in Figure 2. The SMG model is defined in Section 3. Demand response constraints ensure the welfare of a group of users and the essential supply of energy. The 0-1 KP or DRF method is formulated to ensure the aforementioned restrictions, and are defined in Sections 4.1 and 4.2. Finally, the results are verified by comparing the traditional 0-1 KP method with the DRF.

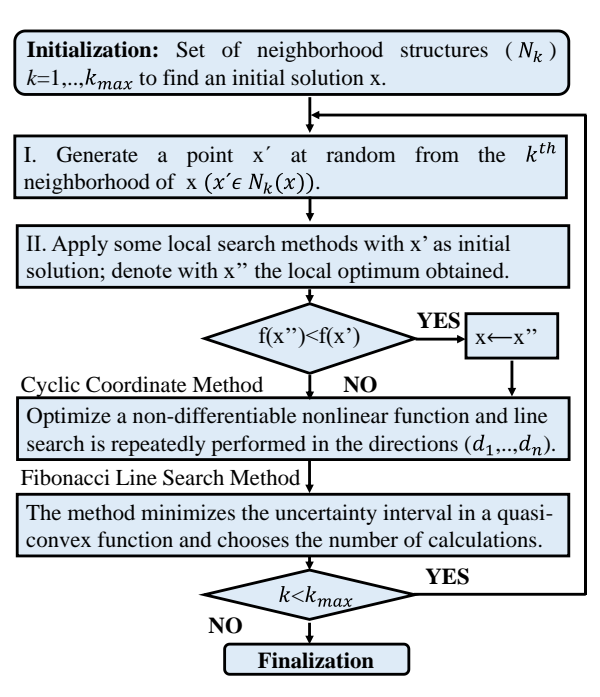

(a)

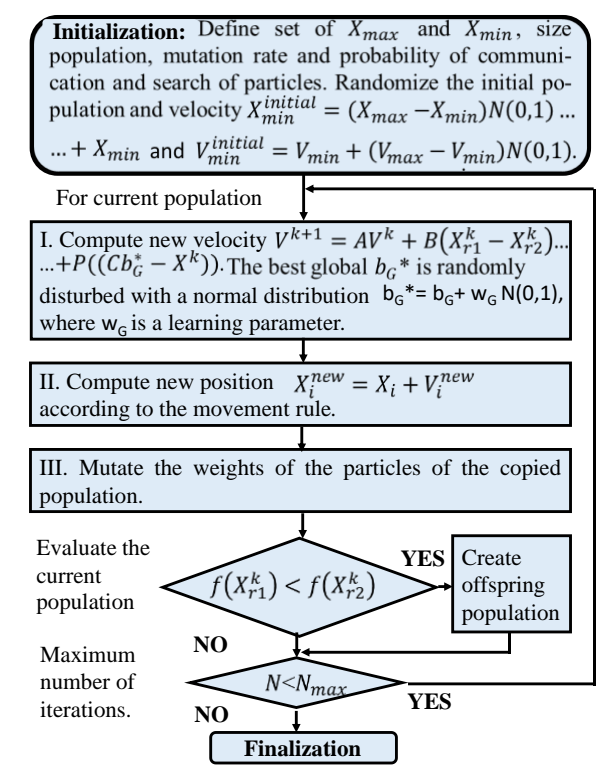

(b)

Figure 1. Algorithm flowchart: (a) variable neighborhood search (VNS); (b) differential evolutionary particle swarm optimization (DEEPSO). Code in detail available: http://www.gecad.isep.ipp.pt/ WCCI2018-SG-COMPETITION/. 


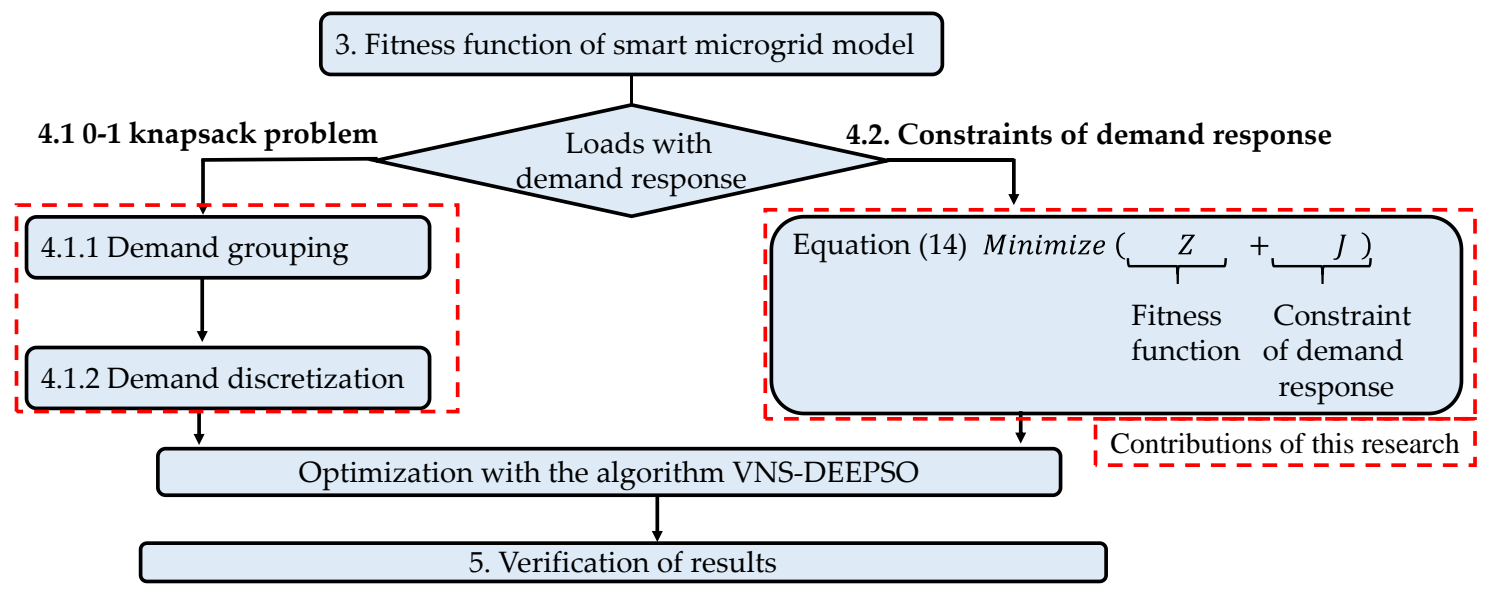

Figure 2. Conceptual formulation of a smart microgrid (SMG) with DR constraints.

\section{Smart Microgrid Model}

The aggregator aims to improve the SMG profits in the day-ahead operation $($ Day +1$)$ [30]. Day-ahead scheduling of residential microgrid is considered an optimization problem [21]; this microgrid located in Portugal includes EVs, residential loads, and an energy storage system, as shown in Figure 3 [35]. A SMG scheduling problem contains 17 aggregated $P V$ generators (17 agg.) in a group of PV generation [36]. This SMG model contains different types of variables, such as binary, continuous, and discrete, by using mixed integer programming $[16,21]$. The aggregator reduces the operational costs (OCs) and maximizes the incomes (In), as shown in Equation (1) [21].

$$
\text { Minimize } \mathrm{Z}=O \mathrm{OC}_{\text {Total }}^{\text {Day+1 }}-\operatorname{In}_{\text {Total }}^{\text {Day+1 }}
$$

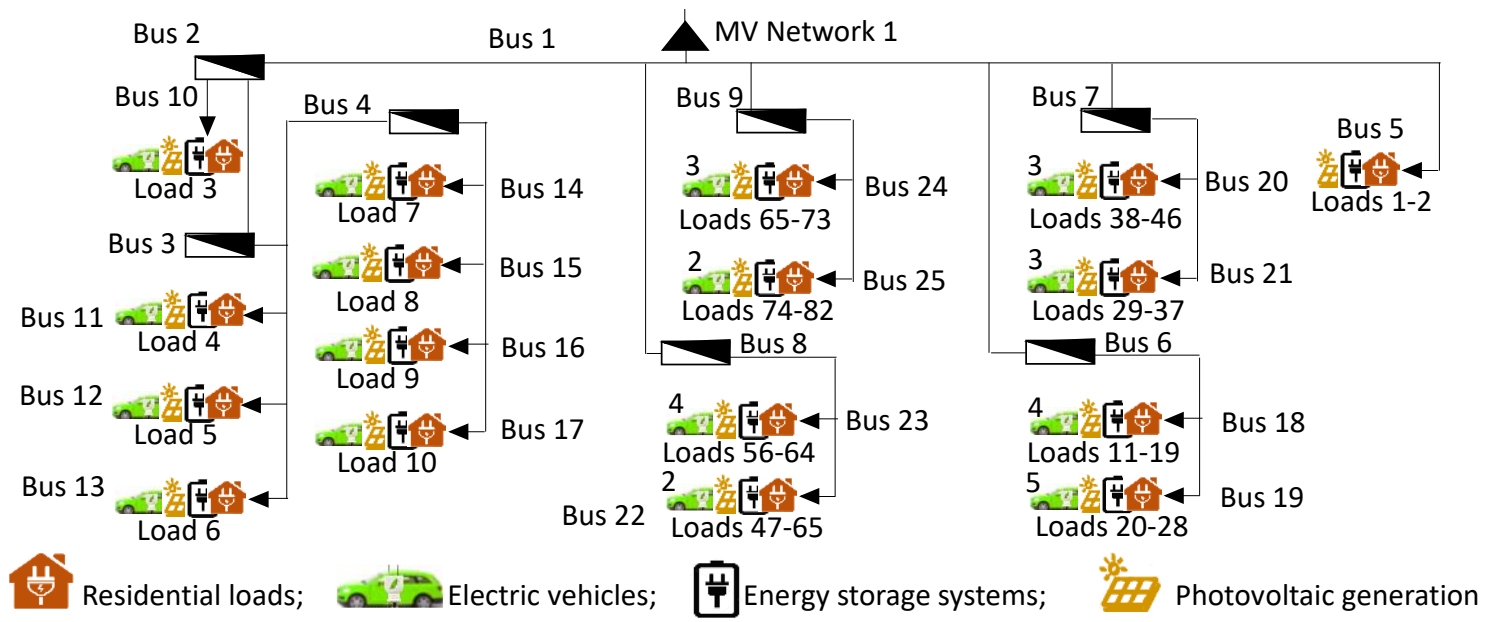

Figure 3. Residential 25-bus SMG in a $400 \mathrm{~V}$ system [35].

The OCs are associated to DGs, ESSs, external supplier (ext), EVs, $P V$ generation, negative $\left(i m b^{-}\right.$) and positive $\left(i m b^{+}\right)$imbalance by exceeding the generation and shortage of energy, and curtailable loads with residential $D R$ (curt), as shown in Equation (2) [13,17]. The OCs have a scenarios distribution probability $\pi(s)$ and predict the forecast error through Monte Carlo simulations [21]. These simulations are based on historical data [16]. The scenarios are reduced with the Soares technique, which is based on statistical metrics. Scenarios are reduced from 5000 to 100 feasible for $P V$ generation, load, 
and market prices [37]. An EVs simulation tool is employed to generate the travel route forecast [38]. Table 3 shows the specifications of the microgrid.

$$
O C_{\text {Total }}^{\text {Day+1 }}=\sum_{s=1}^{N_{S}} \sum_{t=1}^{T}\left(\begin{array}{c}
\sum_{t=1}^{T} \sum_{i=1}^{N_{D G}} P_{D G(i, t)} \cdot C_{D G(i, t)}+\sum_{t=1}^{T} \sum_{k=1}^{N_{k}} P_{e x t(i, t)} \cdot C_{e x t(i, t)}+\ldots \\
\sum_{j=1}^{N_{P V-D G}} P_{P V(j, t, s)} \cdot C_{P V(j, t, s)}+\sum_{e=1}^{N_{e}} P_{E S S^{-}(e, t, s)} \cdot C_{E S S^{-}(e, t, s)} \ldots \\
\ldots+\sum_{v=1}^{N_{v}} P_{E V^{-}(v, t, s)} \cdot C_{E V^{-}(v, t)}+\sum_{l=1}^{N_{L}} P_{\text {curt }(l, t, s)} \cdot C_{c u r t}(l, t, s) \ldots \\
\sum_{l=1}^{N_{L}} P_{i m b^{-}(m, t, s)} \cdot C_{i m b^{-}(m, t, s)}+\sum_{i=1}^{N_{D G}} P_{i m b^{+}(m, t, s)} \cdot C_{i m b^{+}(m, t, s)}
\end{array}\right) \cdot \pi(s)
$$

Table 3. Specifications of SMG [16].

\begin{tabular}{cccc}
\hline SMG Energy Resources & Capacity (kW) & $\begin{array}{c}\text { Prices } \\
(\mathbf{m . u} . / \mathbf{k W})\end{array}$ & Units \\
\hline DGs & $10-100$ & $0.07-0.11$ & 5 \\
External supplier & $0-150$ & $0.074-0.16$ & 1 \\
Charge/discharge of ESSs & $0-16.6$ & 0.03 & 2 \\
Charge/discharge of EVs & $0-111$ & 0.06 & 34 \\
Loads with DR & $4.06-8.95$ & 0.0375 & 90 \\
Wholesale/local market & $0-100 / 10$ & $0.021-0.039$ & 1 \\
\hline & Forecast (kW) & & 1 (17 agg.) \\
\hline PV generation & $0-106.81$ & - & 90 \\
Load & $35.82-83.39$ & - & \\
\hline
\end{tabular}

The aggregator increases the incomes by selling and buying energy in the wholesale and local markets, as shown in Equation (3).

$$
\operatorname{In}_{\text {Total }}^{\text {Day }+1}=\sum_{s^{\prime}=1}^{N_{S}} \sum_{t=1}^{T}\left(\sum_{m=1}^{N_{m}}\left(P_{\text {buy }(m, t)}-P_{\text {sell }(m, t)}\right) \cdot M P_{(m, t, s)}\right) \cdot \pi(s)
$$

The major constraint is to maintain the active power balance, which includes the sum of $P V$ generation, ESS, DG, external supplier, energy imbalance, load curtailment, buy/sale of energy markets, and $E V s$, as shown, respectively, in Equation (4). The generation, ESS, EVs, external supplier, and DR curtailment are limited for each period $t$, as shown in Equations (5)-(9). Binary variables $X$ represent the on/off state for each element. Equation (1) is subject to:

$$
\begin{aligned}
& \sum_{j=1}^{N_{P V-D G}} P_{P V(j, t, s)}+\sum_{e=1}^{N_{e}}\left(P_{E S S^{-}(e, t, s)}-P_{E S S^{+}(e, t, s)}\right)+\sum_{i=1}^{N_{D G}} P_{D G(i, s)}+\sum_{k=1}^{N_{K}} P_{e x t(k, t)}+\sum_{i=1}^{N_{D G}} P_{i m b^{+}(i, t, s)} \\
& \left.\ldots+\sum_{l=1}^{N_{L}} P_{\text {imb }}(l, t, s)\right)+\sum_{l=1}^{N_{L}}\left(P_{\text {curt }(l, t, s)}-P_{\text {load }(l, t, s))}+\sum_{m=1}^{N_{m}}\left(P_{\text {buy }(m, t)}-P_{\text {sell }(m, t, s)}\right)\right. \\
& \cdots+\sum_{v=1}^{N_{v}}\left(P_{E V^{-}(v, t, s)}-P_{E V^{+}(v, t, s)}\right)=0 \quad \forall t \in T, \forall s \in S, \\
& P_{D G \min (i, t)} X_{D G(i, t)} \leq P_{D G(i, t)} \leq P_{D G \max (i, t)} X_{D G(i, t)} \forall t \in T, \forall i \in N_{D G} \\
& P_{\text {ext }(k, t)} \leq P_{\text {extmax }(k, t)} X_{\text {ext }(k, t)} \quad \forall t \in T, \forall k \in N_{k} \\
& P_{\operatorname{cur}(l, t, s)} \leq P_{\operatorname{curtmax}(l, t, s)} X_{\operatorname{curt}(l, t, s)} \quad \forall t \in T, \forall l \in L, \forall s \in S \\
& P_{E S S \min (e, t, s)} X_{E S S(e, t, s)} \leq P_{E S S(e, t, s)} \leq P_{\operatorname{ESSmax}(i, t)} X_{E S S(e, t, s)} \quad \forall t \in T, e \in N_{e}, \forall s \in S
\end{aligned}
$$




$$
\begin{gathered}
P_{E V \min (v, t, s)} X_{E V(v, t, s)} \leq P_{E V(v, t, s)} \leq P_{E V \max (v, t, s)} X_{E V(v, t, s)} \quad \forall t \in T, \forall v \in N_{v}, \forall s \in S \\
e, i, v, k, l \in \mathbb{Z} \quad \forall e \in N_{e}, \forall i \in N_{D G}, \forall v \in N_{v}, \forall k \in N_{k}, \forall l \in L
\end{gathered}
$$

The maximum number of evaluations of the objective function in Equation (1) is limited to 50,000, as shown in Equation (10). This is calculated by multiplying the number of populations, scenarios, and iterations in Equation (10) [16].

$$
\text { NFEs }=N_{P} * N_{s} * N_{\text {iteration }} \text {. }
$$

The $R I$ is calculated with the average of the sum of the mean and the standard deviation divided into the number of runs $\left(N_{\text {runs }}\right)$ of the objective function [13].

$$
R I=\frac{1}{N_{\text {runs }}} \sum_{i=1}^{N_{\text {runs }}}(\mu(Z)+\sigma(Z))
$$

\section{Formulation of the 0-1 KP and the DRF}

Sections 4.1 and 4.2 present the formulation of $0-1 \mathrm{KP}$ and DRF. Table 4 summarizes the implementation of the $0-1 \mathrm{KP}$ and DRF formulations that can be carried out using the simulation general framework in the Matlab R2019b program. The minimum demand is scheduled either using the step $2 \mathrm{a}$ or $2 \mathrm{~b}$ of Table 4 .

Table 4. Simulation general framework.

\begin{tabular}{ccc}
\hline No. & Step & Source \\
\hline 1 & Download the case study VNS-DEEPSO & Data ${ }^{1}[19]$ \\
$2 \mathrm{a}$ & Replace 0-1 KP demand groups in search space limits & Section 4.1 \\
$2 \mathrm{~b}$ & Add DRF in fitness function of VNS2.m and DEEPSO_RE.m & Section 4.2 \\
3 & Analysis of results using Send2Organizer.mat, benchmark_Fitness.txt, & Section 3 \\
& benchmark_Summary.txt, and benchmark_Time.txt & \\
\hline & 1 http://www.gecad.isep.ipp.pt/WCCI2018-SG-COMPETITION/ data from GECAD group.
\end{tabular}

\subsection{Formulation of 0-1 Knapsack Problem 0-1 KP}

SMGs are vulnerable to energy shortages; therefore, it is essential to address the problem of power outages to increase their reliability. $0-1 \mathrm{KP}$ is widely used to solve this problem based on combinatorial optimization. The loads of customer are discretized [3]. 0-1 KP is represented by the evaluation of an objective function that is subject to a constraint. Equation (1) is subject to Equation (12).

$$
\begin{gathered}
\sum_{s=1}^{N_{s}} \sum_{t=1}^{T} \sum_{l=1}^{N_{L}} P_{\text {curt }(l, t, s)} \cdot C_{\text {curt }(l, t, s)} \cdot y_{n} \cdot \pi(s) \geq \\
\max \left(\sum_{s=1}^{N_{s}} \sum_{t=1}^{T} \sum_{l=1}^{N_{L}} P_{\operatorname{curt}(l, t, s)} \cdot C_{\operatorname{curt}(l, t, s)} \cdot \frac{\pi(s)}{4}\right) \\
\forall t \in T, \forall l \in L, \forall s \in S \quad y_{n} \in\{0,1\}(n \in\{1,2, \ldots, N\})
\end{gathered}
$$

The problem in Equation (1) is extended by adding a constraint called 0-1 KP. The same analogy for a knapsack applies to Equation (12). The knapsack has a fixed capacity to carry products. The capacity of the knapsack is $25 \%$ of the maximum demand which is fixed at the convenience of the microgrid, and the products that fill the knapsack are stochastic scenarios, time periods, and loads. In other words, a good state of the demand is ensured, given that the reduction of the demand varies from $25 \%$ to $100 \%$ of the maximum demand. The off and on state is represented by the variable $y_{n}$ as 0 and 1 . The number of discrete variables is represented by $N$. The problem is addressed in two stages for better 
compression and is explained in the following subsections. First, the demand is grouped according to similar patterns and, second, the demand is disaggregated into discrete variables.

\subsubsection{Demand Grouping}

Figure 4 represents the load forecast with the generated scenarios which are represented by demand profiles. Despite uncertainty in the demand forecast, the main trends are presented. In the afternoon, the maximum loads are expected during the day, and at 11 p.m. at night, between 1 and 2 p.m. [16]. The demand falls smoothly from 1 a.m. to 6 a.m., with a peak over $41 \mathrm{~kW}$ at 4 a.m. The load gradually increases from 7 a.m. at midday and it progressively decreases from 4 p.m. to 7 p.m.

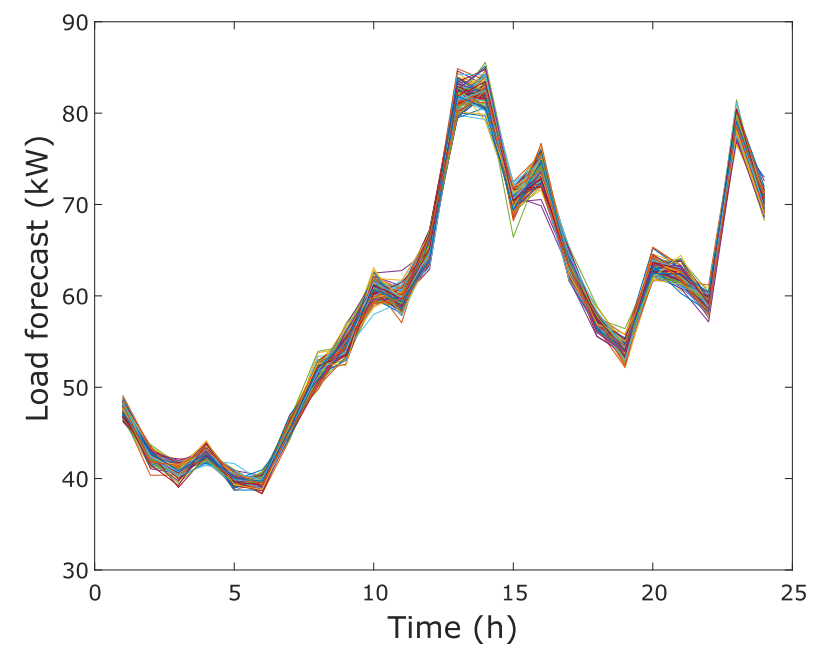

Figure 4. Load forecast for $24 \mathrm{~h}$.

Groups of loads are stacked to the maximum loads of 90 users, as shown in Figure 5a. The loads are classified into three groups with similar characteristics with the colors red, blue, and green. Figure $5 \mathrm{~b}$ shows the selected groups according to the similarity of their demand.

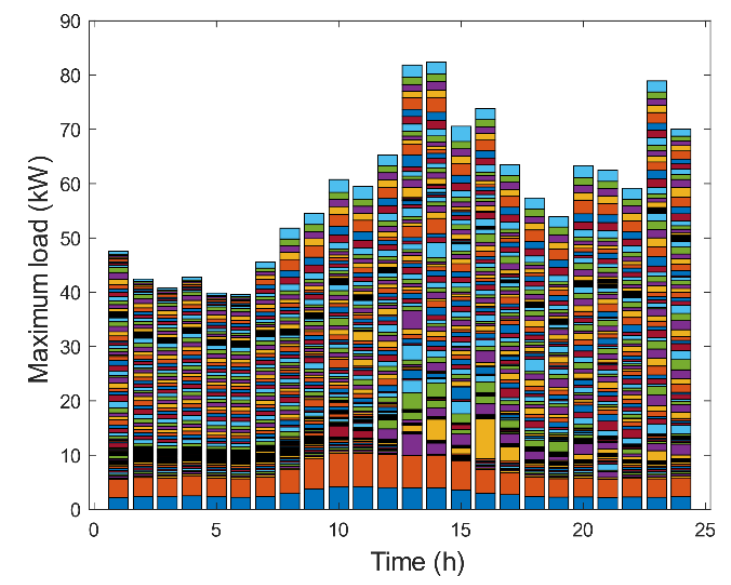

(a)

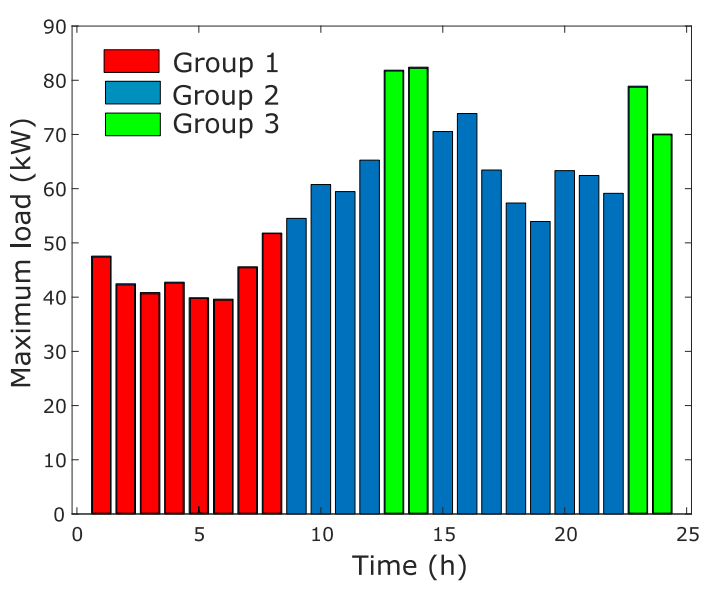

(b)

Figure 5. Maximum demand: (a) stacked load of 90 users; (b) selection of groups for loading with DR.

\subsubsection{Demand Discretization}

The mean is calculated for groups 1, 2, and 3, shown in Figure 5b. These values are 43.8, 62.01, and $78.31 \mathrm{~kW}$, respectively. The groups are subdivided according to these values. The purpose is to convert continuous variables into discrete variables. Discrete groups should have similar sizes in terms 
of means. Under this condition, groups 1, 2, and 3 are subdivided into 2, 3, and 4, respectively. Then, 68 discrete variables are obtained for a 24-h period, as shown in Figure 6.

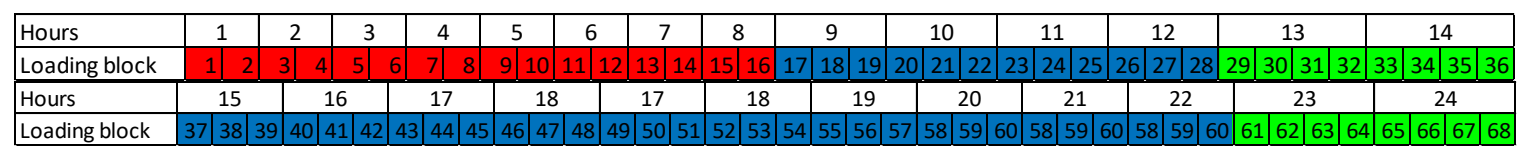

Figure 6. Load forecast for $24 \mathrm{~h}$ in the SMG.

In the next step, the number of combinations is calculated for a load of $25 \%$ which is selected for demand convenience. The calculation is represented with $N_{d}=68$ and $n_{d}=17$, using Equation (13). The variables comprise an array of binary variables of $68 \times 409.29$ quintillion. In the Matlab R2019b program, the maximum variable size is exceeded, so it does not work with this matrix size. The computer processor is Core i5-7200-12 GB RAM. The next combination is generated by taking $N_{d}=34$ and $n_{d}=9$ with a matrix around $34 \times 1715$ million variables and by using Equation (13). The maximum variable size is exceeded again. The next combination is $N_{d}=17$ and $n_{d}=5$ that results in $17 \times 127,858$. With this matrix size, optimization is feasible.

$$
\sum_{n_{d}}^{N_{d}}\left(\frac{N_{d} !}{\left(N_{d}-n_{d}\right)}\right)=N_{C}
$$

The 24-h period is discretized into 17 loads, as shown in Figure 7. One of the concerns of this problem is polynomial nondeterministic time, better known as the N-P hard problem. The polynomial increase is evident [8]. The initial formulation with 68 discrete charges requires a matrix of the order of quintillion. The half of the discrete loads (34) requires a matrix of millions of discrete variables. The half of the discrete loads (17) requires an array of thousands of discrete variables. In other words, the main concern in this type of combinatorial problem is the polynomial calculation time. In 0-1 $\mathrm{KP}$, additional computation times are avoided by preloading a binary matrix of $17 \times 127,858$ with all possible solutions. This matrix is represented by the symbol $y_{n}$, as shown in Equation (12).

\begin{tabular}{|l|c|c|c|c|c|c|c|c|c|c|c|c|c|c|c|c|c|c|c|c|c|c|r|r|}
\hline Hours & 1 & 2 & 3 & 4 & 5 & 6 & 7 & 8 & 9 & 10 & 11 & 12 & 13 & 14 & 15 & 16 & 17 & 18 & 19 & 20 & 21 & 22 & 23 & 24 \\
\hline Loading block & 1 & 2 & 3 & 4 & 5 & 6 & 7 & 8 & 9 & 10 & 11 & 12 & 13 & 14 & 15 & 16 & 17 \\
\hline
\end{tabular}

Figure 7. Discretization in 17 loads in the SMG.

\subsection{Formulation of Demand Response Function (DRF)}

DRF is formulated based on the analogy of actual SMGs. In other words, if a group of users runs out of energy, then, the aggregator will receive a financial penalty. This penalty is received in the objective function, as shown in Equation (14).

$$
\text { Minimize }(Z+J)
$$

where $Z$ represents the objective function and $J$ represents the penalty. This ensures the welfare of the demand. The objective function is detailed in Equation (1); the penalization is given in monetary units (m.u.). The penalization is represented in Equation (15).

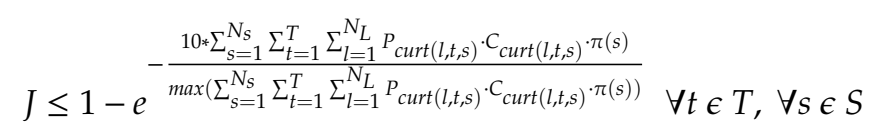

This formulation is motivated by the transfer function in the first order systems [39] and yields results like those presented in Figure 8 [39]. The system output is represented by $J$ and the stabilization time $\left(T^{\prime}\right)$ is represented by the maximum load. It is worth noting that the load is fixed by taking 
fractions of maximum load. In the early phase, there is a steep slope. Next, the slope gradually falls and finishes with the fixed value of the load, represented in blue; this means that it is equivalent to the capacity in 0-1 KP.

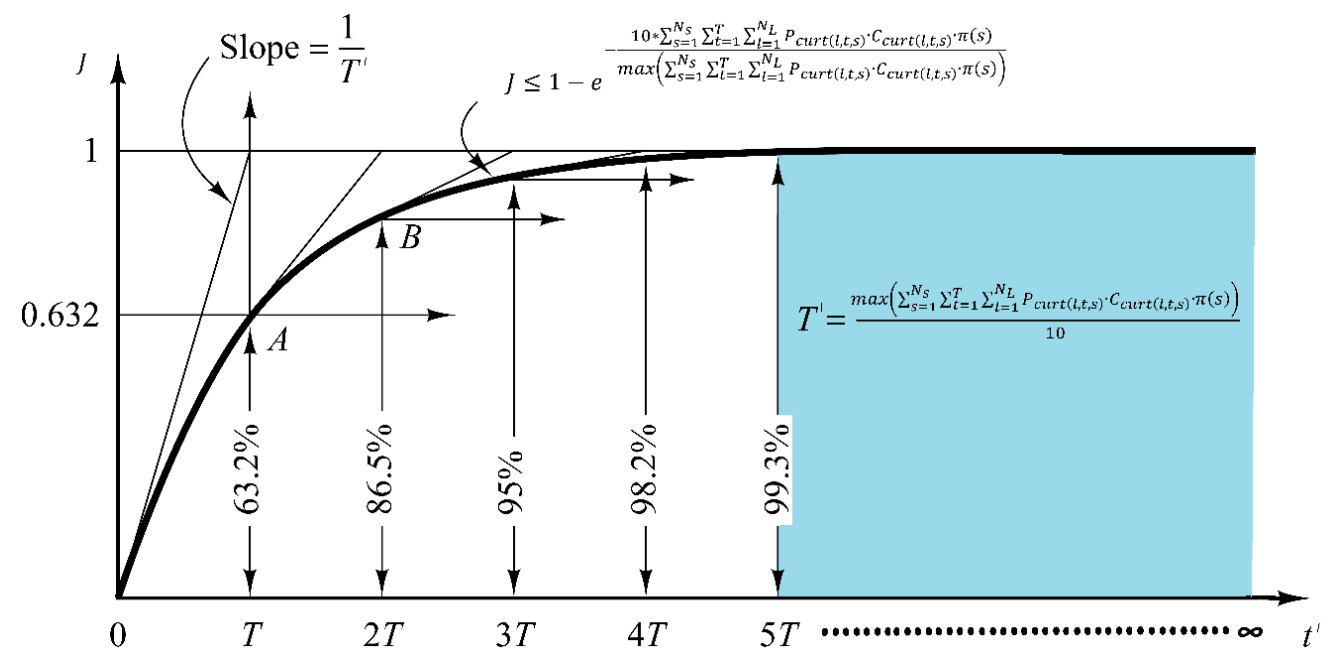

Figure 8. Exponential response curve for the first order system [40].

\section{Results and Discussion}

VNS-DEEPSO was selected based on the review of heuristic optimization algorithms $[15,16,19]$. This algorithm was proposed to solve $0-1 \mathrm{KP}$ and DRF. In fact, the profits were optimized for the SMG model in Equation (1). This case study was optimized for 100 scenarios and 50,000 evaluations of objective function, as shown in Equation (10) [36]. Figure 9 summarizes the load curtailment with DR in percentage. The two methodologies are labeled as (Figure 9a) 0-1 KP and (Figure 9b) DRF. The blue area represents the percentage of load curtailment and the orange area represents the percentage of load shedding. The sum of the orange and blue area must be $100 \%$, which represents the demand forecast for 90 residential loads. The load reduction with 0-1 KP drops from 20\% to 5\%, from 1 to 9 a.m. The load curtailment fluctuates between $5 \%$ and $18.33 \%$, from 10 a.m., at 3 p.m., and in the remaining hours of the day, the load disconnection increases from $5 \%$ to $95 \%$ with slight fluctuations, as shown in Figure 9a. The DRF solution reaches a peak of $66.87 \%$ load reduction during the first $4 \mathrm{~h}$ and the remainder of the day the load shedding is almost constant between $21.67 \%$ and $22.12 \%$, as shown in Figure 9b.

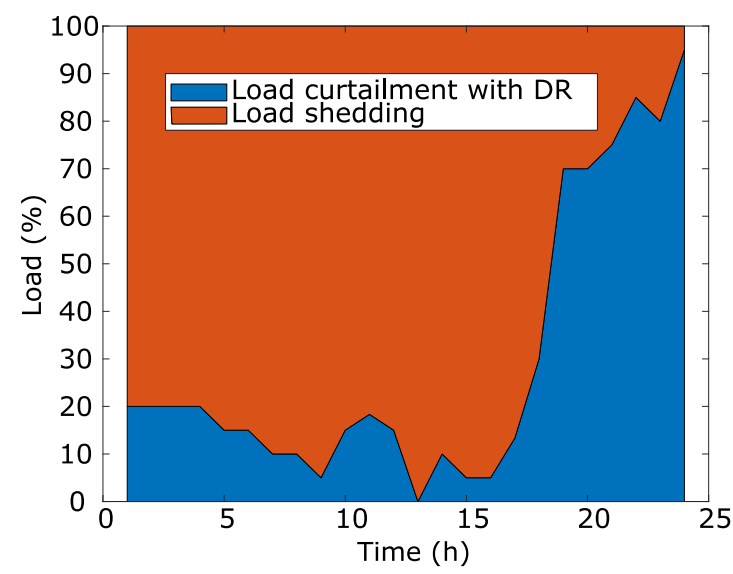

(a)

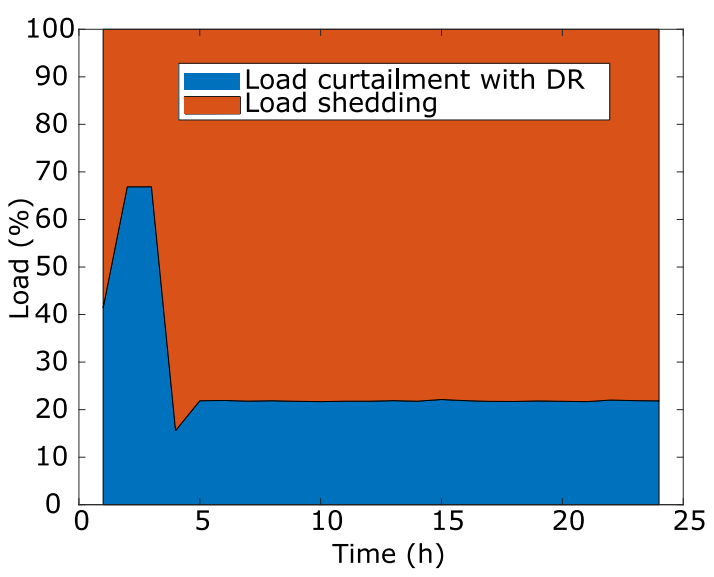

(b)

Figure 9. Load profile with DR: (a) 0-1 knapsack problem; (b) demand response function. 
Figure 10 shows DR for all consumers. The solution with 0-1 KP and DRF are labelled with (a) and (b), respectively. The load profile of 0-1 KP reveals the following patterns. The load with modestly decreases from around 10 to $3 \mathrm{~kW}$ in the period between 1 a.m. and 9 a.m. A mild plateau takes place around $10 \mathrm{~kW}$ between 10 and 12 p.m. Load demand is substantially eliminated at 1 p.m. Between 2 and 3 p.m., there is a slight increase in the load, which does not last long and gradually increases to a peak of around $68 \mathrm{~kW}$. The profile using the DRF presents the following trends, as shown in Figure 10b. The major consumptions occur between 1 a.m. and 3 a.m. Demand consumption increases smoothly from 7 to $18 \mathrm{~kW}$ between $4 \mathrm{a} . \mathrm{m}$. and 1 p.m. Three decreasing slopes are repeated for the periods between 4 p.m. and 7 p.m., between 8 a.m. and 10 p.m., and between 11 p.m. and 12 a.m. Finally, the 0-1 KP solution has sharper peaks compared to DRF. To sum up, 0-1 KP disconnects the load without following a clear pattern, whereas in DRF the loads are disconnected from 4 a.m. until the end of the day. DRF gently follows the trends of the demand forecast and suggests a substantive use of the microgrid between 1 and 3 a.m. DRF is presumed to follow a smooth trend with the use of continuous variables, unlike $0-1 \mathrm{KP}$ with discrete variables.

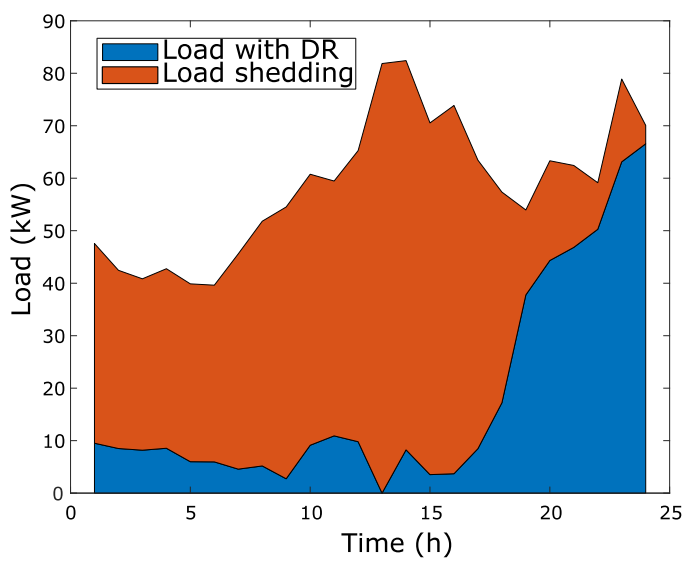

(a)

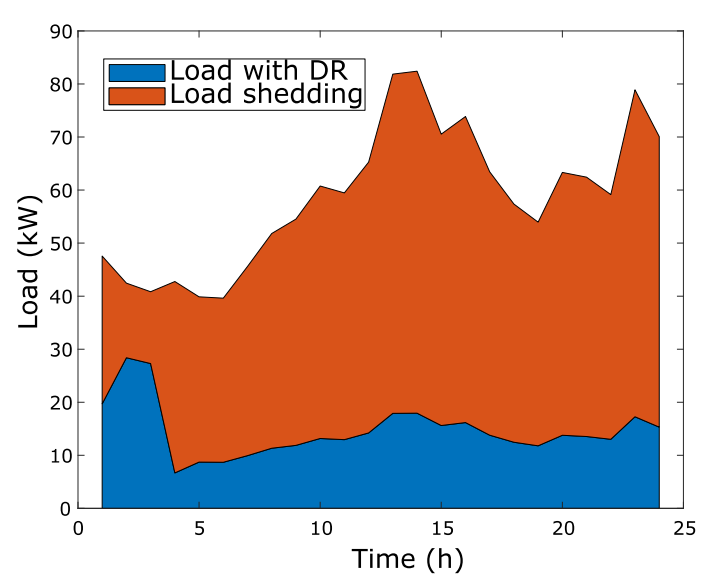

(b)

Figure 10. Demand response for all customers: (a) 0-1 knapsack problem; (b) demand response function.

Figure 11a shows the RI for 20 runs with 1-0 KP, variability in the solutions, and unfavorable benefits in runs $6,7,17$, and 18 . In contrast, DRF keeps the RI fairly stable. In addition, the average $\mathrm{RI}$ is better for DRF with $32.8 \mathrm{~m} . \mathrm{u}$. than for 0-1 KP with $38.5 \mathrm{~m}$.u. with an average improvement of 5.7 m.u. in the 20 runs. Figure $11 \mathrm{~b}$ shows reduction percentage of load shedding with DR. 0-1 KP has three runs with sharp peaks in runs 3,6 , and 18 , and a slight spike in run 17 . The remaining runs are stable. In addition, DRF has an outstanding peak in run 16 . The remaining runs yield consistent values. The average percentage of DR shedding is 31\%, $2 \%$ for $0-1 \mathrm{KP}$, and $25 \%$ for DRF.

Figure 12a shows the execution time. 0-1 KP has favorable execution times, as a result of preloading files with binary combinations. Slight peaks in the running times occur in runs 11, 15, and 18. DRF shows uniform stability in the solution, except for run 6. In general, the execution times are very close for both methods. Figure $12 \mathrm{~b}$ shows the generation divided into DGs and PV generation. DGs have reduced participation with 0-1 KP, whereas DGs with DRF actively participate with smooth fluctuations around $130 \mathrm{~kW}$ during the day. The PV generation has a higher participation than in the DGs with 0-1 KP. The opposite case happens with DRF. In addition, the PV generation with DRF injects more power into the SMG than 0-1 KP. The crunch point generation is presented for 1 and 4 p.m. 


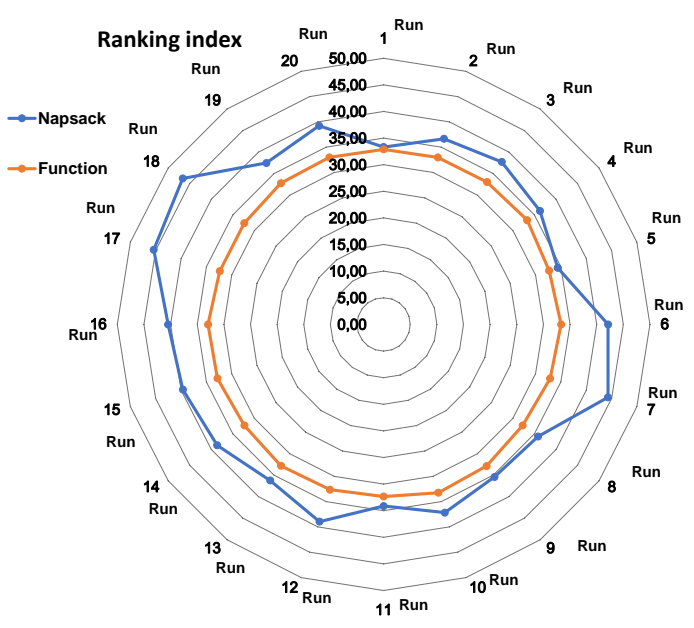

(a)

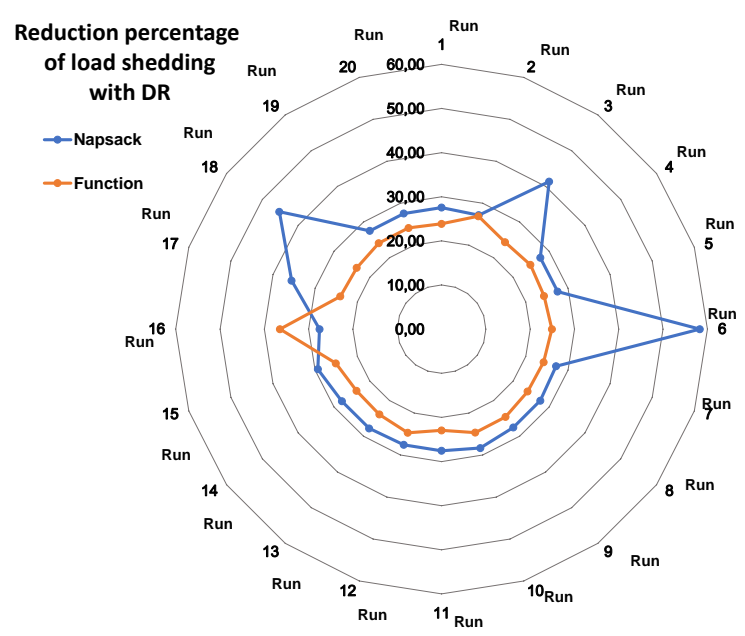

(b)

Figure 11. Analysis of performance for 20 runs: (a) ranking index; (b) reduction percentage of load shedding.

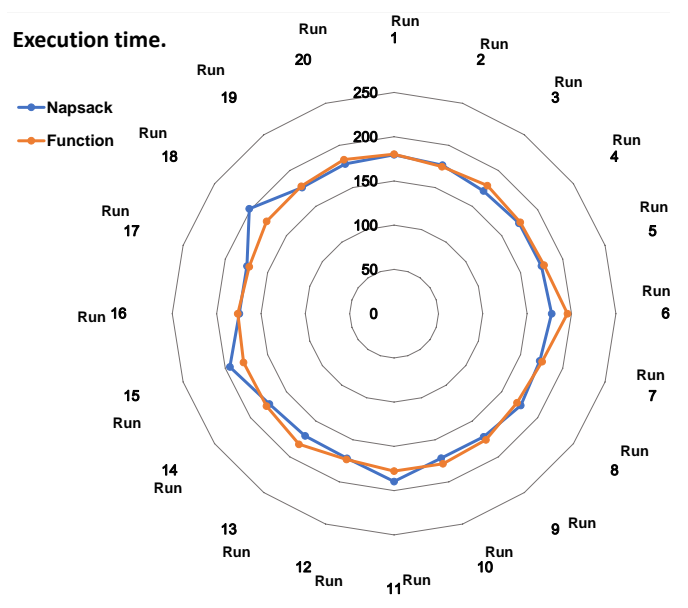

(a)

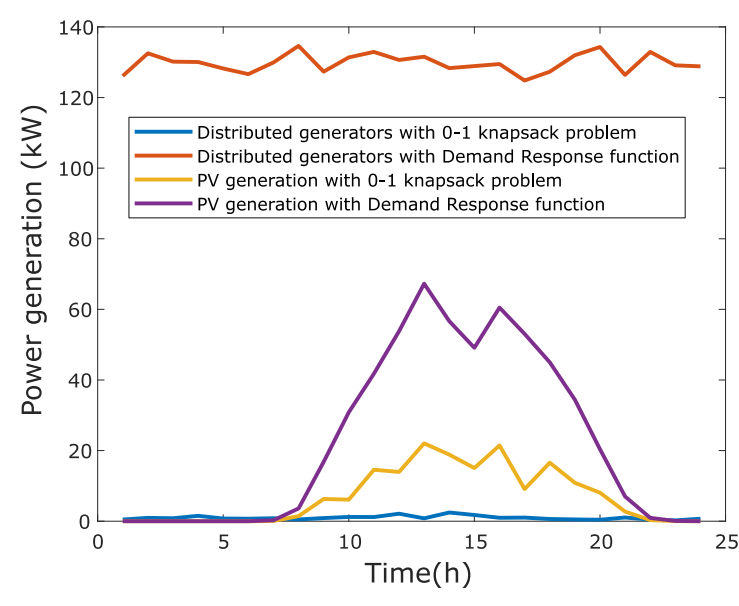

(b)

Figure 12. Results with the $0-1 \mathrm{KP}$ and the DRF. (a) Execution time for 20 runs; (b) power exchanged by the electric vehicles (EVs) and the energy storage systems (ESSs).

Energy is transferred to the grid with ESSs and EVs, as shown in Figure 13a. The ESSs with DRF consume more energy during the first $3 \mathrm{~h}$, then decrease to a smooth consumption of roughly 7 $\mathrm{kW}$, whereas the peak power supply of ESSs with 0-1 KP occur at $3 \mathrm{a} . \mathrm{m}$. and power consumption at midday. EVs have low participation with 0-1 KP and their participation is further reduced with DRF. Transfers in electricity markets are represented by a wholesale market and a local market, as shown in Figure 13b. In general terms, 0-1 KP solution has broad support from the electricity markets with notable fluctuations. In contrast, DRF is more moderate in the use of markets and maintains smooth energy consumption.

\section{Discussion of This Paper}

The optimal solution to reduce operating costs is to disconnect the largest number of users. This means that the aggregator reduces network costs by disconnecting as many loads as possible. However, essential and critical loads cannot be disconnected, therefore, this methodology turns out to be non-viable. On the one hand, the DRF maintains the supply of energy penalizing the non-supply of essential loads. In other words, the penalty has an objective opposite to the objective function. This penalty is similar to the criteria for formulating multi-objective optimization problems. On the 
other hand, 0-1 KP does not penalize the objective function, however, it has the disadvantage that it must calculate the complete number of feasible solutions, this calculation can be obtained from the combination of feasible solutions, however, because the execution time grows in polynomial way obtaining optimal solutions in acceptable times is an NP-hard problem. This means that as the discretization of variables increases, the simulation times grow significantly. The 0-1 KP limitations refer to the high simulation times of NP-hard problems. 0-1 KP can be approached with two methods. The first method consists of calculating the combination that would combine in the optimization problem, however, the simulation times are increased in a polynomial way. The second method is implemented in this research and consists of preloading the feasible solutions, the polynomial times are eliminated as evidenced in the results of this research, however, the computer's RAM memory can be saturated as revealed in Section 4.1. The DRF has the limitation that its scope must be tested in other studies, for example, by changing economic parameters over time and calculating the stabilization time for different percentages of load shedding. However, the percentage of energy supply is satisfied satisfactorily for the optimization problem addressed in this study.

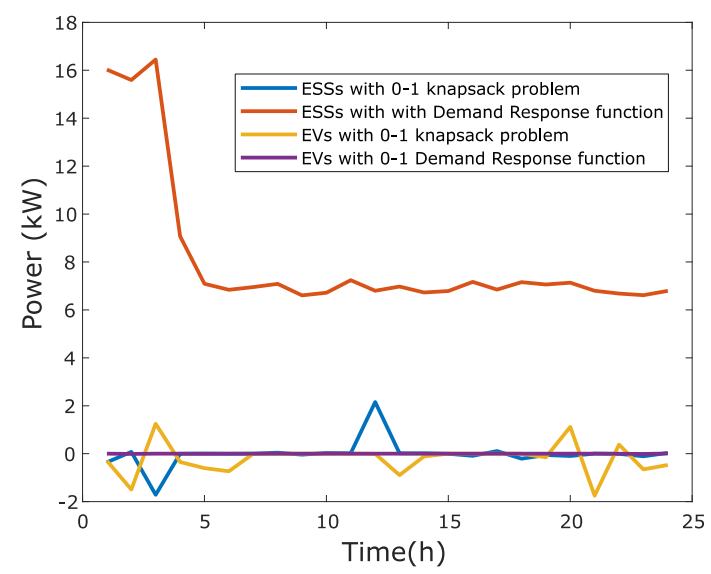

(a)

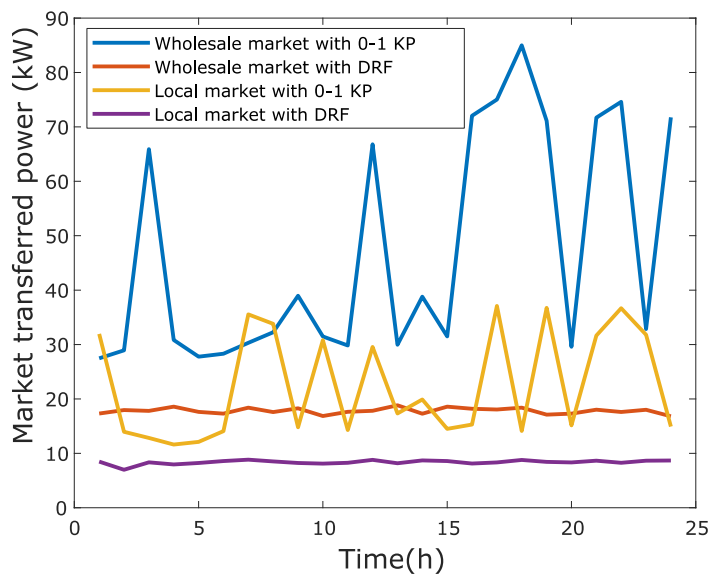

(b)

Figure 13. Power exchanged with 0-1 KP and DRF. (a) EVs and ESSs scheduling for day-ahead; (b) electricity markets scheduling for day-ahead.

\section{Conclusions}

Demand management has benefits for SMGs that include load curtailment and shedding. Optimal participation relieves critical congestion periods. These participation strategies are carried out in a SMG model. The aggregator can manage SMG with a suitable optimization tool, called the VNS-DEEPSO algorithm for loads with DR. This paper proposes to ensure the power supply with the 0-1 KP and DRF method. The 0-1 KP method addresses all feasible solutions in the solution space. Feasible solutions include supplying essential loads. The DRF method penalizes not supplying power. According to the results obtained, $0-1 \mathrm{KP}$ and DRF aim to ensure a minimum supply of energy demand. The classical 0-1 KP method yields outstanding results in terms of load curtailment and shedding. Furthermore, $0-1 \mathrm{KP}$ maintains a reduction with fluctuations around $31.2 \%$ in stochastic scenarios. The alternative constraint with DRF is compared with 0-1 KP and their results are allowable with $25 \%$ load curtailment. Therefore, the restrictions are satisfied satisfactorily for the investigated case study.

DRF has the following advantages regarding 0-1 KP. (1) DRF requires no discretization of variables. In fact, DRF works with continuous variables that yield more accurate outcomes. (2) DRF requires no preload of a file with the combinatorial solution. In other words, 0-1 KP does not make it possible to refine the load blocks at the user's will. Refinement of load blocks depends on the capacity of the computer. (3) DRF needs no additional execution time. This polynomial time appears when 0-1 KP generates the combinatorial sequence. (4) DRF has the best RI. (5) DRF is easier to implement than 
0-1 KP. Finally, some trends are identified for suboptimal planning of DGs, PV generation, EVs, ESSs, and electricity markets.

In future research, the $0-1 \mathrm{KP}$ can be explored in optimization problems whose loads can be grouped and the loads can be represented by discrete variables. Additionally, for future applications, DRF can be further explored for optimization problems without adding loads. That is, the loads are analyzed individually, and a penalty ensures the power supply. In the future, the DRF should be analyzed with other percentages of load reduction and in real SMGs that host residential loads.

Author Contributions: The present work brings together projects of the Universidad Nacional de Colombia and University of Florida with J.G.-G., D.A., S.R., and A.B.; J.G.-G. is supported by Colciencias called 785 for national doctorates. This research was carried out under the supervision, analysis, and preparation of D.A., S.R., and A.B., the preparation of the original draft and formulation was undertaken by J.G.-G.; the review and editing were undertaken by, D.A., S.R., and A.B.; funding acquisition by S.R. and A.B.; finally, project administration was carried out by S.R. All authors have read and agreed to the published version of the manuscript.

Funding: This research received no external funding.

Conflicts of Interest: The authors declare no conflict of interest.

\section{Nomenclature}

\section{Index}

i DG units

$j \quad$ PV units

$k \quad$ External suppliers

$e \quad$ ESSs

$v \quad$ EVs

$l \quad$ Loads

$m \quad$ Markets

$s \quad$ Scenarios

$t \quad$ Periods

\section{Subscript}

DG Distribution generation

PV Photovoltaic

P Populations

K External suppliers

$e \quad$ ESSs

$v \quad$ EVs

L Loads

m Markets

$s \quad$ Scenarios

ext $\quad$ External supplied (kW)

ESS- Discharge ESS (kW)

EV- Discharge EV $(\mathrm{kW})$

ESS+ Charge ESS (kW)

$\mathrm{EV}+\quad$ Charge EV $(\mathrm{kW})$

curt Reduction of load (kW)

Imb- Non-supplied for load $(\mathrm{kW})$

$\mathrm{Imb}+\quad$ Exceeded of DG unit $(\mathrm{kW})$

buy Buy from the market $(\mathrm{kW})$

sell Sell to the market $(\mathrm{kW})$

min Minimum

max Maximum

\section{Variables with Greek letters}

$\pi(s) \quad$ Probability of scenario $s$

$\mu(Z) \quad$ Mean of fitness function

$\sigma(Z) \quad$ Deviation standard of fitness function 


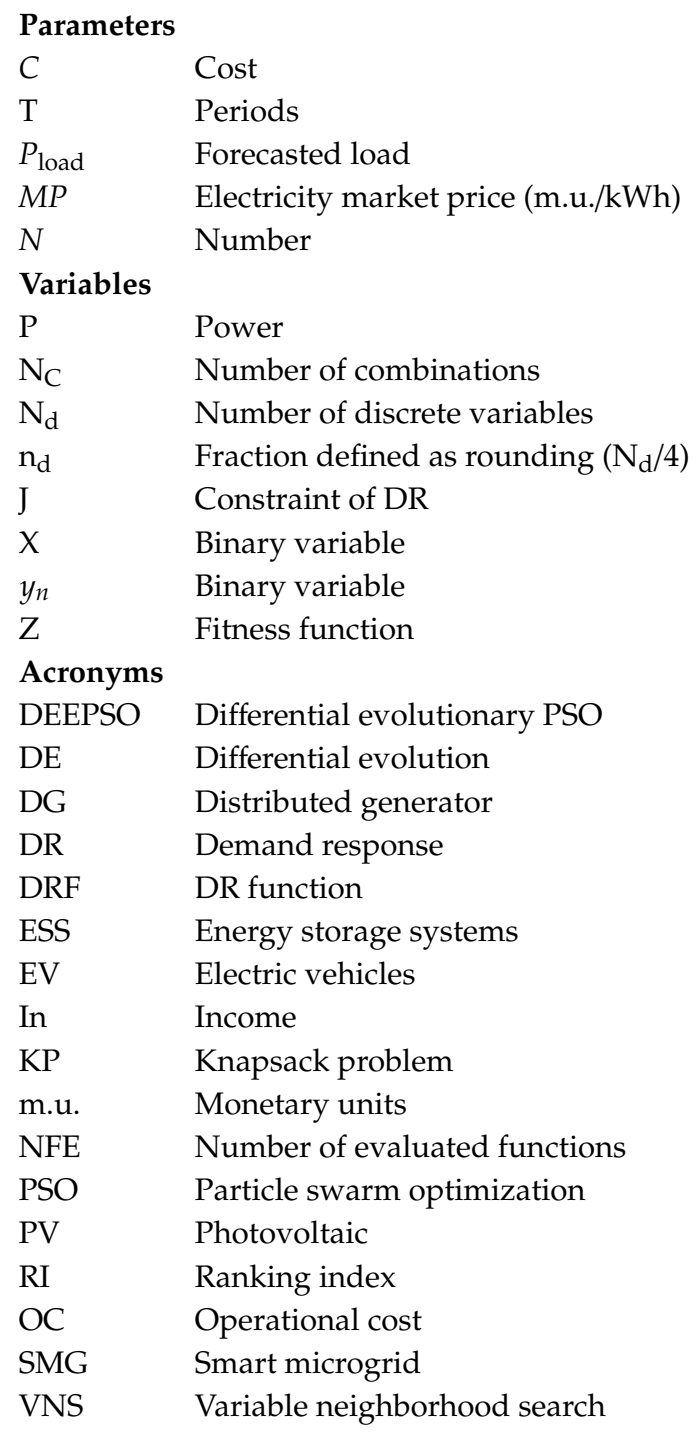

\section{References}

1. Garcia-Guarin, J.; Rivera, S.; Rodriguez, H. Smart grid review: Reality in Colombia and expectations. J. Phys. Conf. Ser. 2019, 1257, 012011. [CrossRef]

2. Dickerson, W.; Goldstein, A.; Kirkham, H.; Martin, K.; Roscoe, A.; De Vries, R.; Wright, P. Smart grid measurement uncertainty: Definitional and influence quantity considerations. In Proceedings of the 2018 1st International Colloquium on Smart Grid Metrology, SmaGriMet 2018, Split, Croatia, 24-27 April 2018; pp. $1-5$.

3. Choi, S.; Park, S.; Kim, H.M. The application of the 0-1 knapsack problem to the load-shedding problem in microgrid operation. In Communications in Computer and Information Science (CCIS); Springer: Berlin/Heidelberg, Germany, 2011; Volume 256, pp. 227-234.

4. Garcia-Guarin, J.; Rivera, S.; Trigos, L. Multiobjective optimization of smart grids considering market power. J. Phys. Conf. Ser. 2019, 1409, 012006. [CrossRef]

5. Pavas Martinez, F.A.; Gonzalez Vivas, O.A.; Sanchez Rosas, Y.S. Cuantificación del ahorro de energía eléctrica en clientes residenciales mediante acciones de gestión de demanda. Rev. UIS Ing. 2017, 16, 217-226. [CrossRef]

6. Cruz, L.M.; Alvarez, D.L.; Al-Sumaiti, A.S.; Rivera, S. Load Curtailment Optimization Using the PSO Algorithm for Enhancing the Reliability of Distribution Networks. Energies 2020, 13, 3236. [CrossRef]

7. Garcia, J.; Alvarez, D.; Rivera, S. Ensemble Based Optimization for Electric Demand Forecast: Genetic Programming and Heuristic Algorithms. Rev. Int. Métodos Numér. Cálc. Diseño Ing. 2020, 1, 1-13. [CrossRef] 
8. Sapre, M.S.; Patel, H.; Vaishnani, K.; Thaker, R.; Shastri, A.S. Solution to small size 0-1 knapsack problem using cohort intelligence with educated approach. In Studies in Computational Intelligence; Springer: Berlin/Heidelberg, Germany, 2019; Volume 828, pp. 137-149.

9. Garcia-Guarin, J.; Duran-Pinzón, M.; Paez-Arango, J.; Rivera, S. Energy planning for aquaponics production considering intraday markets. Arch. Electr. Eng. 2020, 69, 89-100. [CrossRef]

10. Azad, M.A.K.; Rocha, A.M.A.C.; Fernandes, E.M.G.P. A simplified binary artificial fish swarm algorithm for 0-1 quadratic knapsack problems. J. Comput. Appl. Math. 2014, 259, 897-904. [CrossRef]

11. Layeb, A. A hybrid quantum inspired harmony search algorithm for 0-1 optimization problems. J. Comput. Appl. Math. 2013, 253, 14-25. [CrossRef]

12. Xu, Y.; Liu, W. Novel multiagent based load restoration algorithm for microgrids. IEEE Trans. Smart Grid 2011, 2, 140-149. [CrossRef]

13. Carvalho, J.P.P.; Shafie-Khah, M.; Osorio, G.; Rokrok, E.; Catalao, J.P.S. Multi-agent system for renewable based microgrid restoration. In Proceedings of the 2018 International Conference on Smart Energy Systems and Technologies (SEST 2018), Sevilla, Spain, 10-12 September 2018; pp. 1-6.

14. Cheng, Y.; Zhang, C. Configuration and operation combined optimization for EV battery swapping station considering PV consumption bundling. Prot. Control Mod. Power Syst. 2017, 2, 26. [CrossRef]

15. Garcia-Guarin, J.; Rodriguez, D.; Alvarez, D.; Rivera, S.; Cortes, C.; Guzman, A.; Bretas, A.; Aguero, J.R.; Bretas, N. Smart microgrids operation considering a variable neighborhood search: The differential evolutionary particle swarm optimization algorithm. Energies 2019, 12, 3149. [CrossRef]

16. Lezama, F.; Soares, J.; Vale, Z.; Rueda, J.; Wagner, M. CEC/GECCO 2019 Competition Evolutionary Computation in Uncertain Environments: A Smart Grid Application, Rio de Janeiro, Brasil, July, 2018. Available online: https://www.gecad.isep.ipp.pt/ERM2019-Competition/files/CEC_GECCO_2019_ Guidelines_CISG.pdf (accessed on 13 July 2020).

17. Molina Sanchez, F.S.; Pérez Sichacá, S.J.; Rivera Rodriguez, S.R. Formulación de Funciones de Costo de Incertidumbre en Pequeñas Centrales Hidroeléctricas dentro de una Microgrid. Ing. USBMed 2017, 8, $29-36$. [CrossRef]

18. Sanchez, A.; Romero, A.; Rattá, G.; Rivera, S. Smart charging of PEVs to reduce the power transformer loss of life. In Proceedings of the 2017 IEEE PES Innovative Smart Grid Technologies Conference-Latin America, ISGT Latin America 2017, Quito, Ecuador, 20-22 September 2017; Volume 2017, pp. 1-6.

19. García-Guarín, P.J.; Cantor-López, J.; Cortés-Guerrero, C.; Guzmán-Pardo, M.A.; Rivera, S. Implementación del algoritmo VNS-DEEPSO para el despacho de energía en redes distribuidas inteligentes. INGE CUC 2019, 15, 142-154. [CrossRef]

20. Narimani, M.R.; Azizivahed, A.; Naderi, E. An Efficient Scenario-based Stochastic Energy Management of Distribution Networks with Distributed Generation, PV Module, and Energy Storage. arXiv 2019, arXiv:1910.07109.

21. Lezama, F.; Soares, J.; Faia, R.; Pinto, T.; Vale, Z. A New Hybrid-Adaptive Differential Evolution for a Smart Grid Application under Uncertainty. In Proceedings of the 2018 IEEE Congress on Evolutionary Computation, CEC 2018, Rio de Janeiro, Brazil, 8-13 July 2018; pp. 1-8.

22. Garcia-Guarin, J.; Infante, W.; Ma, J.; Alvarez, D.; Rivera, S. Optimal Scheduling of Smart Microgrids Considering Electric Vehicle Battery Swapping Stations. Int. J. Electr. Comput. Eng. 2020, 10, 5093-5107.

23. Chen, J.; Yang, B.; Guan, X. Optimal demand response scheduling with Stackelberg game approach under load uncertainty for smart grid. In Proceedings of the 2012 IEEE Third International Conference on Smart Grid Communications, SmartGridComm 2012, Tainan, Taiwan, 5-8 November 2012; Volume 1, pp. 546-551. [CrossRef]

24. Matthiss, B.; Momenifarahaniy, A.; Ohnmeissz, K.; Felderx, M. Influence of Demand and Generation Uncertainty on the Operational Efficiency of Smart Grids. In Proceedings of the 7th International IEEE Conference on Renewable Energy Research and Applications (ICRERA 2018), Paris, France, 14-17 October 2018; pp. 751-756.

25. Di Somma, M.; Graditi, G.; Heydarian-Forushani, E.; Shafie-khah, M.; Siano, P. Stochastic optimal scheduling of distributed energy resources with renewables considering economic and environmental aspects. Renew. Energy 2018, 116, 272-287. [CrossRef]

26. Saber, A.Y.; Venayagamoorthy, G.K. Resource scheduling under uncertainty in a smart grid with renewables and plug-in vehicles. IEEE Syst. J. 2012, 6, 103-109. [CrossRef] 
27. Radhakrishnan, B.M.; Srinivasan, D.; Mehta, R. Fuzzy-Based Multi-Agent System for Distributed Energy Management in Smart Grids. Int. J. Uncertain. Fuzziness Knowl. Based Syst. 2016, 24, 781-803. [CrossRef]

28. Parhizi, S.; Khodaei, A.; Shahidehpour, M. Market-based versus price-based microgrid optimal scheduling. IEEE Trans. Smart Grid 2018, 9, 615-623. [CrossRef]

29. Farzan, F.; Jafari, M.; Masiello, R.; Lu, Y. Toward optimal day-ahead scheduling and operation control of microgrids under uncertainty. IEEE Trans. Smart Grid 2015, 6, 499-507. [CrossRef]

30. Garcia Guarin, J.; Lezama, F.; Soares, J.; Rivera, S. Operation scheduling of smart grids considering stochastic uncertainty modelling. Far East J. Math. Sci. 2019, 1, 77-98. [CrossRef]

31. Naderi, E.; Azizivahed, A.; Narimani, H.; Fathi, M.; Narimani, M.R. A comprehensive study of practical economic dispatch problems by a new hybrid evolutionary algorithm. Appl. Soft Comput. J. 2017, 61, 1186-1206. [CrossRef]

32. Fossati, J.P. Revisión bibliográfica sobre micro redes inteligentes. Lit. Rev. Microgrids 2011, 9, 13-20.

33. De Vargas Fortes, E.; Macedo, L.H.; de Araujo, P.B.; Romero, R. A VNS algorithm for the design of supplementary damping controllers for small-signal stability analysis. Int. J. Electr. Power Energy Syst. 2018, 94, 41-56. [CrossRef]

34. Miranda, V.; Alves, R. Differential Evolutionary Particle Swarm Optimization (DEEPSO): A successful hybrid. In Proceedings of the 1st BRICS Countries Congress on Computational Intelligence (BRICS-CCI 2013), Ipojuca, Brazil, 8-11 September 2013; pp. 368-374.

35. Soares, J.; Silva, M.; Canizes, B.; Vale, Z. MicroGrid der control including EVs in a residential area. In Proceedings of the 2015 IEEE Eindhoven PowerTech (PowerTech 2015), Eindhoven, The Netherlands, 29 June-2 July 2015; Institute of Electrical and Electronics Engineers Inc.: New York, NY, USA, 2015.

36. Lezama, F.; Sucar, L.E.; de Cote, E.M.; Soares, J.; Vale, Z. Differential evolution strategies for large-scale energy resource management in smart grids. In Proceedings of the Genetic and Evolutionary Computation Conference Companionc, Berlin, Germany, 15-17 July 2017; pp. 1279-1286. [CrossRef]

37. Soares, J.; Canizes, B.; Ghazvini, M.A.F.; Vale, Z.; Venayagamoorthy, G.K. Two-Stage Stochastic Model Using Benders' Decomposition for Large-Scale Energy Resource Management in Smart Grids. IEEE Trans. Ind. Appl. 2017, 53, 5905-5914. [CrossRef]

38. Soares, J.; Canizes, B.; Lobo, C.; Vale, Z.; Morais, H. Electric vehicle scenario simulator tool for smart grid operators. Energies 2012, 5, 1881-1899. [CrossRef]

39. Trigos, A.; Garcia-Guarin, J.; Blanco, E. Design of a PID control for a prototype of an automated GMAW welding bench. J. Phys. Conf. Ser. 2019, 1257, 1-9. [CrossRef]

40. Ogata, K. Modern Control Engineering; Prentice Hall: New York, NY, USA, 2002. 